\title{
Metastatic Seminoma Presenting as Enlarging Retroperitoneal Mass in a Patient with Neurofibromatosis I
}

Joseph J LaConti ${ }^{1 *}$, Tae Kon Kim ${ }^{1,4}$, Elizabeth Whittington ${ }^{2}$, Nignyuan Ding ${ }^{1}$, Vy Dinh ${ }^{3}$, Khushman Moh'd ${ }^{3}$, Saleem Umar ${ }^{2}$ and Stefan Glück ${ }^{3}$

${ }^{1}$ Department of Internal Medicine, Jackson Memorial Hospital, University of Miami, Miami, FL, USA

${ }^{2}$ Department of Pathology, Jackson Memorial Hospital, University of Miami, Miami, FL, USA

${ }^{3}$ Sylvester Comprehensive Cancer Center, Division of Hematology/Oncology, Miami, FL, USA

${ }^{4}$ Smilow Cancer Center, Yale University Medical Center, New Haven, CT, USA

\begin{abstract}
Neurofibromatosis type 1 (NF1) is a rare genetic disorder where patients develop neurofibromas and have characteristic skin and eye findings. These patients have increased risks for certain types of cancers, especially of neural origin. Testicular cancer is a relatively common type of cancer for younger men, and is considered a very treatable malignancy if identified early. This report describes a patient with NF1 who had an initial chief complaint of back pain. A retroperitoneal mass was identified, but initial pathology report was epithelioid granulomas without necrosis, and was non-diagnostic. After the patient developed deep vein thrombosis as complication of the mass, a repeat biopsy would provide the diagnosis of typical seminoma. A second review by independent pathologists of the primary biopsy sample was also unable to appropriately diagnose the seminoma. This report illustrates how a clear pathological diagnosis can drastically alter the approach to the disease and change the prognosis in a patient with an already rare disorder.
\end{abstract}

Keywords: Neurofibromatosis; Seminoma; Retroperitoneal mass

\section{Introduction}

Neurofibromatosis type 1 (NF1) is a rare autosomal dominant disorder that affects 1 in 3000 people. It is also known as von Recklinghausen's Disease, named after the German pathologist who first described the syndrome in 1882 . The disorder is caused by a mutation in the NF1 gene on chromosome 17q11.2 that encodes for the neurofibromin protein, a guanosine triphosphatase activating protein that functions as a modulator of RAS signaling. The disease manifests itself with a variety of physical findings, and the diagnosis is made when two or more of the following signs are present: neurofibromas, which are soft, rubbery cutaneous tumors, six or more café-au-lait macules, axillary or inguinal freckling, optic glioma, two or more iris hamartomas (Lisch nodules), sphenoid dysplasia, or an immediate relative with NF1 [1]. These patients also have an increased incidence of chronic myeloid leukemia, neurofibrosarcoma, rhabdomyosarcoma, pheochromocytoma, cerebral astrocytoma, and brainstem glioma [2]. A prospective study of NF1 patients in the UK showed an overall cancer risk increase of 2.7 times the general population, with the most frequent types being of connective tissue and neural origin [3].

Cancer of the testicle is the most common malignancy afflicting men 25 to 40 years of age. Ninety five percent of testicular cancers arise from malignant germ cells, and of these germ cell tumors (GCT) $40-50 \%$ are seminoma type. The presentation of testicular cancer, regardless of type, is typically a painless testicular mass or nodule, with associated swelling [4]. Disease occurs at extragonadal sites in 5-10\% of patient. Retroperitoneal adenopathy can cause back pain, and given the local anatomy of the retroperitoneum, the aorta and ureters can be affected as well [5].

Here we present a case of a 23 year old male with neurofibromatosis found to have a seminoma with metastasis to the retroperitoneum, who had initial symptoms of back pain and developed bilateral deep vein thromboses of the iliac veins and bilateral ureteral compression that required stenting.

\section{Case}

This is a case of a 23 year old male diagnosed with NF1 as a child. He exhibited classical signs of the disease including café-au-lait spots
(Figure 1A), Lisch nodules (Figure 1B), and a history of optic glioma that was surgically removed. The patient initially presented with a complaint of back pain. It was described as a dull ache that did not radiate and was only occasionally alleviated by anti-inflammatory medication. An MRI showed a retroperitoneal mass and a laparoscopic biopsy was planned. However, due to bleeding complications the procedure was converted to an open laparotomy. The pathology on the biopsy specimen was read by an outside hospital as epithelioid granulomas without necrosis, with a broad differential of neoplastic process, lymphoma, autoimmune, or infectious origin. Before the patient could follow up with specialists, he was re-admitted to the hospital with a complaint of swelling in his bilateral lower extremities and continual back pain. Ultrasound Doppler of the lower extremities showed bilateral deep vein thromboses of the right external iliac vein to the proximal superficial femoral vein, and dampened wave forms of the left lower extremity suggestive of a proximal occlusion. A repeat CT scan showed an increase in size of the previously seen retroperitoneal mass. It measured $10 \mathrm{~cm}$, was described as encasing the aorta and compressing the ureters bilaterally (Figure 2). The retroperitoneal mass was biopsied by interventional radiology with CT guidance and pathology on this specimen revealed a small population of malignant cells consistent with seminoma, in a background of lymphocytic infiltrate and cellular necrosis (Figure 3). Immunohistochemistry was positive for Cluster of differentiation 117 (CD117, Ckit) and placental alkaline phosphatase (PLAP) and negative for Transducin-like enhancer protein 1 (TLE1), CD30, keratin, alpha feto-protein (AFP), myogenin, desmin, epithelial membrane antigen (EMA), S100, and human Chorionic Gonadotrophin (hCG). Octamer-binding transcription

*Corresponding author: Joseph J LaConti, Internal Medicine, Jackson Memoria Hospital, Central Building, 600D, 1611 NW 12th St, Miami, FL, 33136, USA, Tel: 732-904-8469; E-mail: jlaconti@med.miami.edu

Received May 24, 2014; Accepted June 28, 2014; Published June 30, 2014

Citation: LaConti JJ, Kim TK, Whittington E, Ding N, Dinh V, et al. (2014) Metastatic Seminoma Presenting as Enlarging Retroperitoneal Mass in a Patient with Neurofibromatosis I. J Clin Case Rep 4: 380. doi:10.4172/2165-7920.1000380

Copyright: @ 2014 LaConti JJ, et al. This is an open-access article distributed under the terms of the Creative Commons Attribution License, which permits unrestricted use, distribution, and reproduction in any medium, provided the original author and source are credited. 


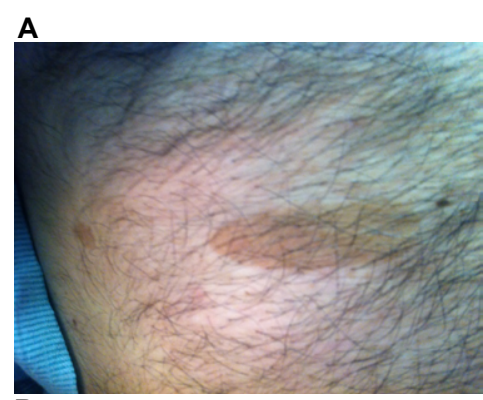

B

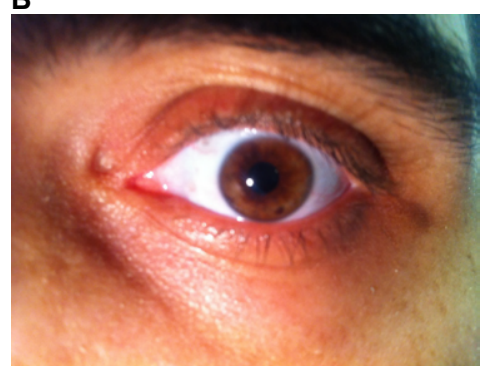

Figure 1: A) café-au-late spots, B) Lisch nodule.
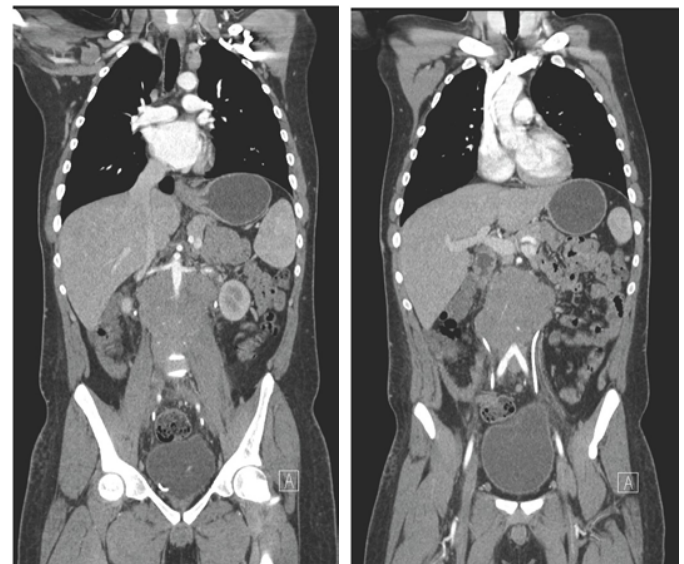

Figure 2: Sagittal CT scan of retroperitonealmass. Left, the mass is seen surrounding the abdominal aorta inferior to the renal arteries. Right, the mass is seen superior to the aortic bifurcation. In the right image, bilateralureteral stents can be seen.

factor 4 (Oct-4) was equivocal. At this point the patient had a scrotal ultrasound which revealed a complex hypervascular, hypoechoic mass in the right testicle, $2 \mathrm{~cm} \times 1.3 \mathrm{~cm} \times 2.5 \mathrm{~cm}$ with micro calcifications. The patient was referred to urology who inserted bilateral 6 French $\mathrm{x} 26 \mathrm{~cm}$ ureteral stents and performed a radical inguinal orchiectomy. The pathology of the testicular specimen showed seminoma, classic type. The tumor invaded the tunica albuginia, with focal involvement of the rete testis, and lymphovascular invasion but the spermatic cord margin and tunica vaginalis were negative. His serological studies showed a lactate dehydrogenase of 978 units/L (normal 313 to 618 units/L), human chorionic gonadotropin of $20.5 \mathrm{mIU} / \mathrm{mL}$, and alpha fetoprotein of $2.7 \mathrm{ng} / \mathrm{mL}$. This tumor was staged as intermediate risk seminoma T2N3M0S2, stage IIIC. Following his surgery he began chemotherapy with a regimen of bleomycin, etoposide, and cisplatin for a total of three cycles. The expected toxicities of this regimen were alopecia, cytopenias, mucositis, and neuropathy, of which the patient only experienced mild mucositis and alopecia. The patient received three cycles but a follow up PET scan showed continued avidity in the retroperitoneal mass. He is currently undergoing targeted radiation to the mass and we look forward to measuring his response to this treatment. The initial pathology report that did not suggest seminoma was also reviewed by the pathology department at our institution, and the initial report of epithelioid granuloma was confirmed.

\section{Discussion}

Here we report a testicular seminoma in a patient with neurofibromatosis 1 that presented as an enlarging retroperitoneal mass. The initial biopsy did not identify seminoma specifically, but instead suggested a wide differential diagnosis. NF1 is associated with an increased risk of malignancy in the breast, brain, and mesenchymal organs but not typically in the gonadal organs [2,3]. In males between the ages of 20-40, cancers of the testicle are the most common types of malignancies [5]. The presentation of this patient's seminoma is not uncommon, as multiple reports show symptoms related to extragonadal disease as the presenting complaint [6]. The differential diagnosis of lower back pain in a male of this age should always include the possibility of a yet to be diagnosed malignancy, including lymphoma, sarcoma, or cancer of testicular origin.

In this case the diagnosis of retroperitoneal mass was made but the pathological diagnosis was not confirmatory. Initially, history and physical was non-contributory in this case. The accurate diagnosis and staging of testicular cancer is paramount, as the prognosis changes significantly with the stage, and testicular cancer is considered one of the most curable types of cancer. The patient's tumor was staged as intermediate risk seminoma T2N3M0S2, stage IIIC. In seminomas, the presence of extra pulmonary metastasis is the defining feature of the intermediate risk group. Despite this connotation, the 5 year survival of any intermediate risk germ cell tumor, whether it be seminoma or nonseminomatous, is $79 \%$ [7].

In a large multicenter analysis of extragonadal seminomas that were either primary mediastinal or primary retroperitoneal, the five year survival rate was $90 \%$ in patients who received platinum based therapy [8]. The same research group generated prognostic variables for survival of a large cohort of extragonadal germ-cell tumors who received platinum based therapy. They reported that the adverse prognostic factors of extragonadal seminomas were liver metastasis, two or greater metastatic sites, or International Germ Cell Cancer Collaborative Group designation of intermediate risk. A very favorable

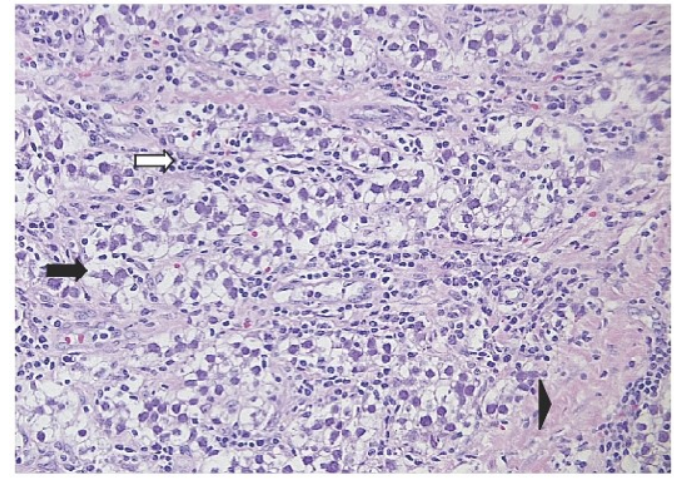

Figure 3: Seminoma, Classic type. Hematoxylin and Eosin stain (20X). Tumor cells are marked by the Black arrow, lymphocytic infiltrate is marked by the white arrow, and a fibrous septae is marked by the arrow head. 
5 year survival of $89 \%$ was found in this group as well, underscoring the benefit of platinum based therapy in seminomas and the improved response rates achieved compared to non-seminomatous pathology [9]. While our patient presented with metastasis to the retroperitoneum, his seminoma tumor type affords him a more favorable prognosis.

The reports of testicular cancer in NF1 patients are few. There is a case report from 1988 that showed a patient with NF1 with a mixed germ cell tumor of the testis [10] and another report from 1990 that showed a unilateral teratoma of the testis in an NF1 patient [11]. A third report described a patient with bilateral testicular tumors that occurred 10 years apart from each other, and were of different pathologies; the first was a right testicular embryonal carcinoma without seminomatous component, and the second a left pure seminoma [12].

However, the increased incidence of malignancy in patients with NF1 has not been definitively linked to the loss of the NF1 gene, and research is still working to determine the involvement of other cellular pathways such as RAS, MAPK, mTOR, and PAK1 in the development of NF1 associated tumors [13]. For testicular cancer, genome studies have suggested a causative gene to be located on the $\mathrm{X}$ chromosome at Xp27, but the gene has yet to be identified $[14,15]$. Despite uncertainty of genetic linkage between NF1 and testicular cancer, our report and others show that there is an association with the two diseases clinically. Further population study will be crucial to demonstrate a definitive relationship, just as NF1 is associated with many other malignancies.

\section{References}

1. Jett K, Friedman JM (2010) Clinical and genetic aspects of neurofibromatosis 1. Genet Med 12: 1-11

2. Seminog OO, Goldacre MJ (2013) Risk of benign tumours of nervous system, and of malignant neoplasms, in people with neurofibromatosis: populationbased record-linkage study. Br J Cancer 108: 193-198.
3. Walker L, Thompson D, Easton D, Ponder B, Ponder M, et al. (2006) A prospective study of neurofibromatosis type 1 cancer incidence in the $\mathrm{UK} . \mathrm{Br} J$ Cancer 95: 233-238.

4. Horwich A, Shipley J, Huddart R (2006) Testicular germ-cell cancer. Lancet 367: 754-765.

5. Horwich A, Bajorin D (1997) Testicular cancer: presentation, assessment and prognosis. Principles and practice of genitourinary oncology. Philadelphia, Lippincott-Raven

6. (2000) Case records of the Massachusetts General Hospital. Weekly clinicopathological exercises. Case 1-2000. A 27-year-old man with a painful retroperitoneal mass. N Engl J Med 342: 115-122.

7. (1997) International Germ Cell Consensus Classification: a prognostic factorbased staging system for metastatic germ cell cancers. International Germ Cell Cancer Collaborative Group. J Clin Oncol 15: 594-603.

8. Bokemeyer C, Droz JP, Horwich A, Gerl A, Fossa SD, et al. (2001) Extragonadal seminoma: an international multicenter analysis of prognostic factors and long term treatment outcome. Cancer 91: 1394-1401.

9. Hartmann JT, Nichols CR, Droz JP, Horwich A, Gerl A, et al. (2002) Prognostic variables for response and outcome in patients with extragonadal germ-cell tumors. Ann Oncol 13: 1017-1028.

10. Groot-Loonen JJ, VoÃ»te PA, de Kraker J (1988) Testicular tumor concomitant with von Recklinghausen's disease. Med Pediatr Oncol 16: 116-117.

11. Hilton DA, Muller S, Macpherson DS (1990) Testicular teratoma and peripheral neurofibromatosis. Postgrad Med J 66: 974-975.

12. Kume H, Tachikawa T, Teramoto S, Isurugi K, Kitamura T (2001) Bilateral testicular tumour in neurofibromatosis type 1. Lancet 357: 395-396.

13. Brems H, Beert E, de Ravel T, Legius E (2009) Mechanisms in the pathogenesis of malignant tumours in neurofibromatosis type 1. Lancet Oncol 10: 508-515.

14. Lutke Holzik MF, Rapley EA, Hoekstra HJ, Sleijfer DT, Nolte IM, et al. (2004) Genetic predisposition to testicular germ-cell tumours. Lancet Oncol 5: 363371.

15. Rapley E (2007) Susceptibility alleles for testicular germ cell tumour: a review. Int J Androl 30: 242-250. 